\title{
Mucopolysaccharidosis type VII presenting as neonatal cholestasis
}

\author{
Reshma Manayankath', Shanavas Abbas², Sankar V Hariharan ${ }^{3}$ \\ From ${ }^{1}$ Assistant Professor, Department of Paediatrics, Government Medical College, Thiruvananthapuram, Kerala, India, ${ }^{2}$ Professor, Department \\ of Paediatrics, Government Medical College, Thiruvananthapuram, Kerala, India, ${ }^{3}$ Additional Professor, Department of Paediatrics, Government \\ Medical College, Thiruvananthapuram, Kerala, India
}

\begin{abstract}
Mucopolysaccharidosis type VII presenting as neonatal cholestasis is very rare. Here, we present the case of a 55-day-old baby presented with cholestatic jaundice. On examination, the baby had icterus, hepatosplenomegaly, and initial workup for common causes of cholestasis which were negative. Liver biopsy revealed neonatal hepatitis. On further follow-up, coarse facies were noted, and suspecting metabolic disorder, exome sequencing was done. A mutation in the GUSB gene was identified which is pathogenic for MPS VII. The neutrophils also showed the characteristic Alder-Reilly granules. Neonatal cholestasis with coarse facies should have MPS VII as a differential diagnosis. Genetic testing in doubtful metabolic conditions will provide an answer and also help in the prenatal diagnosis of future pregnancies.
\end{abstract}

Key words: Cholestasis, Genetic testing, Jaundice, Sly syndrome

$\mathrm{T}$ he mucopolysaccharidoses (MPSs) are inherited lysosomal storage disorders caused by the absence of functional enzymes that help in the degradation of glycosaminoglycans (GAGs). The progressive systemic deposition of GAGs results in multiorgan involvement that varies with the particular GAG deposited and the specific enzyme mutation present. Eleven types of enzyme deficiencies have been described so far and some MPS types have been divided into subtypes according to enzyme defect and the type of GAG eliminated in the urine [1]. MPS type VII, also known as Sly syndrome is a rare lysosomal storage disorder, first reported in 1973 [2]. This syndrome is caused by a deficiency of beta-glucuronidase enzyme due to mutation of the GUSB gene located on chromosome 7q21.11 [1]. In the absence of the enzyme, beta-glucuronidase, dermatan sulfate, chondroitin sulfate, and heparan sulfate will not be degraded and are accumulated in various tissues leading to organ dysfunction.

Sly syndrome is a disorder rarely reported in the literature; especially, from India [3]. The classical presentation varies from severe phenotype characterized by hydrops fetalis, coarse facies, skeletal anomalies, and mental retardation to milder cases with minimal skeletal abnormalities. Neonatal cholestasis is a very rare presentation of Sly syndrome [4]. Here, we report a case of MPS VII who presented with cholestatic jaundice.

\section{Access this article online}

Received - 20 September 2020

Initial Review - 06 October 2020

Accepted - 17 October 2020

DOI: $10.32677 / I J C R .2020 . v 06.110 .016$

\section{CASE REPORT}

A 55-day-old female baby, firstborn of a non-consanguineous marriage, presented with a complaint of jaundice since day 2 of life. She was born out of a cesarean section at 37 weeks, APGAR score at 5 min was 9 , and weighed $2.8 \mathrm{~kg}$. The mother was aged 24 years at the time of conception and she had no significant medical illness. The antenatal period was uneventful except for a third-trimester scan showing a fetal cardiac anomaly with minimal pericardial effusion.

On examination, the baby had icterus, Grade 3/6 systolic murmur, left developmental dysplasia of the hip (DDH), and hepatosplenomegaly (HSM). The baby was exclusively breastfed and was gaining weight adequately.

Initial laboratory investigations revealed a direct hyperbilirubinemia (total/direct:14/4.2 $\mathrm{mg} / \mathrm{dl}$ ), elevated transaminases (serum glutamic-oxaloacetic transaminase/serum glutamic-pyruvic transaminase (SGOT/SGPT)-221/52 IU/L). The baby was worked up for possible causes of neonatal cholestasis which included the TORCH screen, alpha 1 antitrypsin level, urine metabolic screen, and thyroid function tests, all of them were normal. Hepatic scintigraphy showed no evidence of biliary atresia. The ophthalmological evaluation was unremarkable. Echocardiography revealed mitral valve prolapse with mild mitral regurgitation.

Based on these investigations, she was diagnosed as a case of idiopathic neonatal hepatitis and started on supportive

Correspondence to: V H Sankar, Additional Professor, Department of Paediatrics, SATH, Government Medical College Thiruvananthapuram, Kerala, India. E-mail: sankarvh@gmail.com

(C) 2020 Creative Commons Attribution-NonCommercial 4.0 International License (CC BY-NC-ND 4.0). 
measures. She was given prednisolone at $1 \mathrm{mg} / \mathrm{kg} /$ day. Baby was started on 10 times the recommended daily allowances (RDA) of fat-soluble vitamins and two times the RDA of water- soluble vitamins. Liver biopsy done at 3 months of age was suggestive of neonatal hepatitis. Immunohistochemistry (IHC) was done for progressive familial intrahepatic cholestasis (PFIC) 1, 2, and 3 , and the proteins were seen on immunostaining which ruled out the possibility of PFIC.

At 5 months, the baby was readmitted with worsening jaundice and ascites. Physical examination revealed deepening of jaundice, hepatomegaly $4 \mathrm{~cm}$ from the right costal margin, and spleen palpable $8 \mathrm{~cm}$ in the long axis with firm consistency (Fig. 1). The baby had coarse facies, strongly suggestive of a metabolic disorder. However, there were no features of dysostosis multiplex and ophthalmologic findings like corneal clouding. Developmental milestones were age appropriate. Blood investigations revealed total/ direct bilirubin 23/19 mg/dl and SGOT/SGPT 350/117 IU/L. Since there was no definitive clinical diagnosis, exome sequencing was done to look for any rare metabolic disorder causing neonatal hepatitis.

Exome sequencing revealed a homozygous, likely pathogenic mutation in exon 1, c.115C >A (p.Lys39Gln) in the GUSB gene, suggestive of mucopolysaccharidosis type VII (Sly syndrome). Then for confirmation, enzyme estimation in lymphocytes was done which was found to be low. Since Alder-Reilly granules can be demonstrated in this condition, we specifically looked for this and demonstrated in the peripheral blood smear examination (Fig. 2).

Since definitive treatment like stem cell transplantation and enzyme replacement therapy was not affordable to the family, they opted for supportive care for the baby. Genetic counseling was given to the family as the recurrence risk in every pregnancy can be $25 \%$, and prenatal diagnosis can be offered at 12 weeks of pregnancy. The baby expired at the age of 7 months. The mother conceived again after 2 years and the prenatal genetic testing offered by CVS at 12 weeks revealed an unaffected fetus and she delivered a healthy baby boy.

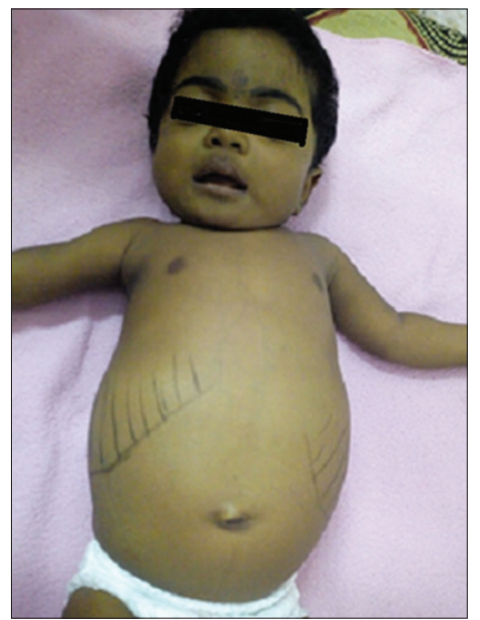

Figure 1: A 5-month-old baby with coarse facies and hepatosplenomegaly

\section{DISCUSSION}

The mucopolysaccharidoses type VII, also called Sly syndrome (OMIM 253220), was first reported by Sly et al. It is inherited as an autosomal recessive condition with gene locus for betaglucuronidase on the long arm of chromosome 7q21.11. The clinical presentation in Sly syndrome is highly variable due to different types of mutations described in the GUSB gene [4].

The classical clinical characteristics were described in a recent large case series [4,5]. The coarse facial feature was present in $87 \%$ of patients. The predominant ocular features were corneal clouding (63\%), heavy eyebrows (52\%), visual impairment (37\%), and photosensitivity (30\%). Recurrent respiratory infection and reduced pulmonary functions were reported in the majority of patients. Heart abnormalities include cardiac valvular disease (50\%) and cardiomyopathies (37\%). Dysostosis multiplex was the most consistent finding (90\%) in the skeletal survey. Other musculoskeletal abnormalities reported are joint contractures/ stiffness, spine, and leg deformities. Developmental delay/mental retardation was the most common neurological manifestation. Another consistent feature was short stature in these cases. Oral manifestations observed in three patients consist of wide root canal spaces, taurodontism, hyperplastic dental follicles, malposition of unerupted permanent molars, and failure of tooth eruption with malformed roots [6].

The most severe form of presentation is lethal non-immune hydrops fetalis (NIHF) and may be detected by the antenatal scan. Our child had a cardiac lesion and pericardial effusion detected in the antenatal period. One characteristic clinical feature that differentiates MPS VII from other MPS is the presence of NIHF in the antenatal period. Recent literature showed the presence of NIHF in $41 \%$ of cases. However, this feature will not predict the eventual severity of the clinical course if the patient survives infancy [4]. Some of the severely affected newborns may survive for some months and develop symptoms of lysosomal storage disorder including thick skin, coarse facies, visceromegaly, and dysostosis multiplex. Less severe forms of MPS VII present during initial years of life with features of MPS I but slower progression.

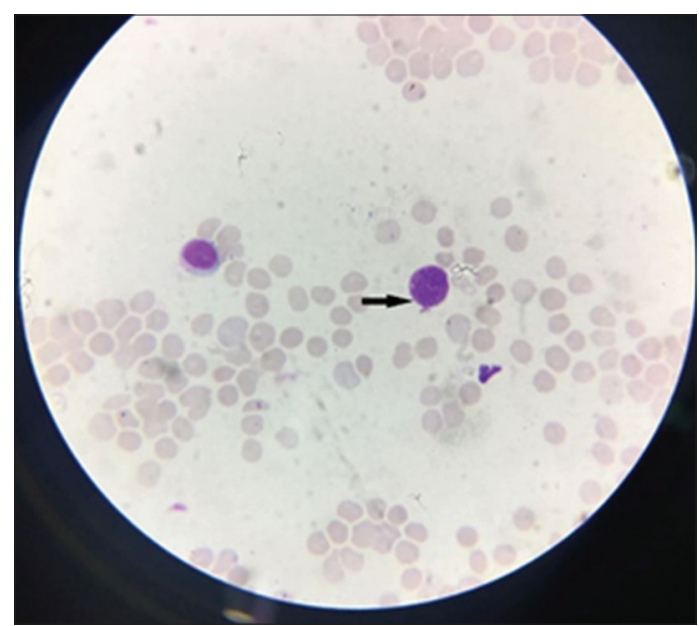

Figure 2: Peripheral smear examination showing Alder-Reilly granules 
Our case had cholestasis as a predominant presentation, which is an unusual manifestation of MPS VII. A similar case has been reported in the literature rarely. A case was reported in 2001 from Canada where the baby presented with neonatal cholestasis, $\mathrm{DDH}$, and was found to have markedly decreased levels of betaglucuronidase activity [7].

The pathogenesis of cholestasis in MPS VII is not known, however, the accumulation of intact and partially degraded glycosaminoglycan material in lysosome may cause distension of hepatocytes and Kupffer cells, resulting in compression of sinusoids and lobular distortion with altered trafficking through the Golgi and lysosome. Furthermore, the enzymes and proteins involved in bile acid transport may be directly inhibited by beta-glucuronidase substrates as there have been no reports of cholestasis in other types of MPS.

Alder-Reilly anomaly is an inherited abnormality of white blood cells associated with mucopolysaccharidosis. In blood smears and bone marrow preparations, the most characteristic finding is the metachromatic granules surrounded by a clear zone seen in lymphocytes. Dense granules, resembling toxic granulation in neutrophils, are seen in all leukocytes. The condition may be mistaken for toxic granulation, a type of abnormal granulation in neutrophils that occur transiently in inflammatory conditions. In addition to mucopolysaccharidosis, Alder-Reilly anomaly may occur in lipofuscinosis and Tay-Sachs disease. Alder-Reilly inclusions stain appears violet when treated with Wright-Giemsa stain and in mucopolysaccharidosis, stain metachromatically with toluidine blue. This is not diagnostic of any disorder and does not correlate with the severity. There is no functional abnormality identified in affected white blood cells. This anomaly is described with the Sly syndrome in previous case reports $[3,8]$. The pathologist should be aware of this when examining the peripheral smear. In our case also, we were able to demonstrate the granules, only when we strongly suspected this.

The definitive diagnosis can be made by reduced or absent beta-glucuronidase activity in granulocyte or cultured skin fibroblast lysosome followed by DNA testing of the specific gene (GUSB). However, in our case, since there was no definitive diagnosis, clinical exome was performed where multiple parallel genes of interest causing neonatal cholestasis were sequenced and enzyme testing was done after the molecular diagnosis. In a study, among the 103 analyzed mutant alleles, missense mutations accounted for $78.6 \%$; non-sense mutations, $12.6 \%$; deletions, $5.8 \%$; and splice site mutations, $2.9 \%$. This heterogeneity in GUSB gene mutations contributes to the extensive clinical variability among patients with MPS VII. The five most frequent mutations accounting for 44/103 alleles were exonic point mutations, p.L176F, p.R357X, p.P408S, p.P415L, and p.A619 V [9]. There is no definite genotype-phenotype correlation observed.

The definitive therapy of MPS type VII is hematopoietic stem cell transplantation (HSCT) and enzyme replacement therapy (ERT). The limited results suggest that HSCT can slow or even prevent further neurological complications but has little or no effect on the skeletal disease unless performed at an early stage [10]. In 2017, vestronidase alfa, an ERT was approved to treat pediatric and adult patients with MPS VII. Given the lack of treatment options and the clinical benefits it provides, intravenous vestronidase alfa is an important emerging ERT for patients with mucopolysaccharidosis VII [11]. Recently, a patient was given combined HSCT and ERT showed better results and outcomes [12]. Novel replacement strategies for MPS VII are in the pipeline. Lentiviral-mediated gene therapy is effective in reversing established skeletal pathology in murine MPS VII [13]. Similarly, animal experiments suggest that fetal therapy with IUERT and/or IUHCT could overcome the shortcomings of current treatment strategies to improve phenotype in MPS VII and other LSDs [14].

\section{CONCLUSION}

MPS VII (Sly syndrome) is a rare heritable disorder of glycosaminoglycan degradation caused by the deficiency of lysosomal enzyme beta-glucuronidase. Hence, a case of neonatal cholestasis with HSM and coarse facies should have MPS VII as a differential diagnosis. This case also demonstrates the use of exome sequencing in the definitive diagnosis of the patient followed by reverse phenotyping. Definitive therapy such as HSCT, ERT, gene therapy, and intrauterine therapy is emerging for this rare genetic disorder. Genetic testing should be done and the mutation when identified in the proband opens the possibility of genetic counseling and prenatal diagnosis in future pregnancies.

\section{REFERENCES}

1. Spranger JW. Mucopolysaccharidoses. In: Kliegman RM, St Geme JW, Blum NJ, Shah SS, Tasker RC, Wilson KM, editors. Nelson Textbook of Pediatrics. $21^{\text {st }}$ ed. Philadelphia, PA: Elsevier; 2020. p. 814.

2. Sly WS, Quinton BA, McAlister WH, Rimoin DL. Beta glucuronidase deficiency: Report of clinical, radiologic, and biochemical features of a new mucopolysaccharidosis. J Pediatr 1973;82:249-57.

3. Nampoothiri S, Mahesh K, Hiran KR, Sunitha V. Sly disease: Mucopolysaccharidosis Type VII. Indian Pediatr 2008;45:859-61.

4. Montaño AM, Lock-Hock N, Steiner RD, Graham BH, Szlago M, Greenstein R, et al. Clinical course of sly syndrome (mucopolysaccharidosis Type VII). J Med Genet 2016;53:403-18.

5. Zielonka M, Garbade SF, Kölker S, Hoffmann GF, Ries M. Quantitative clinical characteristics of 53 patients with MPS VII: A cross-sectional analysis. Genet Med 2017;19:983-8.

6. Kantaputra PN, Smith LJ, Casal ML, Kuptanon C, Chang YC, Nampoothiri S, et al. Oral manifestations in patients and dogs with mucopolysaccharidosis Type VII. Am J Med Genet A 2019;179:486-93.

7. Gillett PM, Schreiber RA, Jevon GP, Israel DM, Warshawski T, Vallance H, et al. Mucopolysaccharidosis Type VII (Sly syndrome) presenting as neonatal cholestasis with hepatosplenomegaly. J Pediatr Gastroenterol Nutr 2001;33:216-20.

8. Huang YL, Li SY, Zhao XY, Liu HS, Ou XB, Liu L. Mucopolysaccharidosis VII: Report of a case and review of the literature. Zhonghua Er Ke Za Zhi 2011;49:455-8.

9. Tomatsu S, Montaño AM, Dung VC, Grubb JH, Sly WS. Mutations and polymorphisms in GUSB gene in mucopolysaccharidosis VII (Sly Syndrome). Hum Mutat 2009;30:511-9.

10. Taylor M, Khan S, Stapleton M, Wang J, Chen J, Wynn R, et al. Hematopoietic stem cell transplantation for mucopolysaccharidoses: Past, present, and future. Biol Blood Marrow Transplant 2019;25:e226-46.

11. McCafferty EH, Scott LJ. Vestronidase alfa: A review in 
mucopolysaccharidosis VII. BioDrugs 2019;33:233-40.

12. Dubot P, Sabourdy F, Plat G, Jubert C, Cancès C, Broué P, et al. First report of a patient with MPS Type VII, due to novel mutations in GUSB, who underwent enzyme replacement and then hematopoietic stem cell transplantation. Int J Mol Sci 2019;20:5345.

13. Derrick-Roberts AL, Panir K, Pyragius CE, Zarrinkalam KH, Atkins GJ, Byers S. Reversal of established bone pathology in MPS VII mice following lentiviral-mediated gene therapy. Mol Genet Metab 2016;119:249-57.

14. Nguyen QH, Witt RG, Wang B, Eikani C, Shea J, Smith LK, et al. Tolerance induction and microglial engraftment after fetal therapy without conditioning in mice with mucopolysaccharidosis Type VII. Sci Transl Med 2020;12:eaay8980.

Funding: None; Conflicts of Interest: None Stated.

How to cite this article: Manayankath R, Abbas S, Hariharan SV. Mucopolysaccharidosis type VII presenting as neonatal cholestasis. Indian J Case Reports. 2020;6(10):594-597. 